\title{
Publisher Correction: Revisiting the gut-joint axis: links between gut inflammation and spondyloarthritis
}

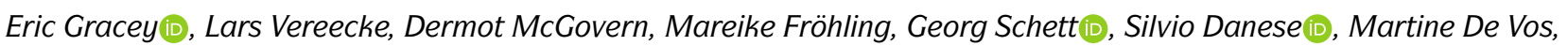
Filip Van den Bosch and Dirk Elewaut (D)

Nature Reviews Rheumatology 16, 415-433 (2020) https://doi.org/10.1038/s41584-020-0454-9 Published online 13 July 2020

In the originally published article, errors in the affiliations have now been corrected in both the HTML and PDF versions of the article, to state: IBD Center, Department of Gastroenterology, Humanitas Clinical and Research Center - IRCCS -, Rozzano, Milan, Italy, and Department of Biomedical Sciences, Humanitas University, Pieve Emanuele, Milan, Italy.

https://doi.org/10.1038/s41584-020-0486-1 I Published online 30 July 2020

(๑) Springer Nature Limited 2020 